
PRAMANA
- journal of
(c) Indian Academy of Sciences

physics

pp. $\quad 1-15$

\title{
The gravitational wave symphony of the Universe
}

\author{
B.S. Sathyaprakash
}

Department of Physics and Astronomy, Cardiff University, Cardiff, CF2 3YB, Great Britain

Abstract. The new millennium will see the upcoming of several ground-based interferometric gravitational wave antennas. Within the next decade a space-based antenna may also begin to observe the distant Universe. These gravitational wave detectors will together operate as a network taking data continuously for several years, watching the transient and continuous phenomena occurring in the deep cores of astronomical objects and dense environs of the early Universe where gravity was extremely strong and highly non-linear. The network will listen to the waves from rapidly spinning non-axisymmetric neutron stars, normal modes of black holes, binary black hole inspiral and merger, phase transitions in the early Universe, quantum fluctuations resulting in a characteristic background in the early Universe. The gravitational wave antennas will open a new window to observe the dark Universe unreachable via other channels of astronomical observations.

Keywords. Gravitational waves, black holes, compact binaries

PACS Nos 04.3.0Db, 04.25.Nx, 04.80.Nn, 95.55.Ym

\section{Introduction}

The next few years will witness the opening of the gravitational window for observing the Universe using a world-wide network of cryogenic resonant bar and kilometer baseline laser interferometric gravitational wave (GW) detectors. I These detectors will take data continuously and will initially be able to observe inspiralling black holes at a distance of $100 \mathrm{Mpc}$ and will enhance their sensitivity by an order of magnitude, and the volume of search by 3 orders of - magnitude, in about five years after they are built, thus increasing the number of potential events a thousand-fold. It is this second phase of operation that will be more interesting from the astrophysical point of view bringing us a new insight into phenomena poorly understood or revealing sources never observed at all. The initial phase, however, may well see the most luminous sources of radiation, such as transient signals arising as a result of the inspiral and merger of black hole binaries and from stellar collapse and supernova explosion. The initial detections, though not expected to be frequent, are important from the fundamental physics point of view and will enable us to directly test predictions of general relativity that have so far eluded all efforts.

We will begin by discussing the nature of GW and their interaction with matter and how that helps in building GW detectors. We will then go on to briefly look at the sensitivities of the upcoming GW antennas and the planned space mission. This will be followed by a detailed discussion of some of the promising sources of GR. For recent reviews on GW see Schutz [], Thorne [], Flanagan [] and Grishchuk et al. []. Lasota and Marck [] and Bhawal and Iyer [] contain many articles on GW sources and their detection.

\section{Gravitational Waves: An Overview}

Gravitational waves result from coherent accelerated motion of massive objects. Any system with a time varying quadrupole moment would emit GW. They interact with particles in their path by causing an oscillating tidal distortion in proper distances between the particles. They are characterized by a dimensionless amplitude $h$. The amplitude $h$ measures the tidal strain $\delta \ell / \ell=h / 2$ in the proper distance between two free particles separated initially by a distance $\ell$. Just as light and radio waves, GW too carry energy and momentum from their sources. Unlike radio waves, however, there is no dipole radiation in Einstein's gravity. The dominant channel for emission is quadrupolar. Since the force of gravity tends to make astronomical objects spherical, or at most axisymmetric, GW emission is an inefficient process, unless the symmetry is broken by non-axisymmetric rotation, presence of magnetic fields, internal stresses due to inhomogeneities, an inherent asymmetry in the system, as in binary star systems, etc.

Sometimes astronomical sources of gravitational radiation (GR) can be very luminous; a supernova explosion at even as great a distance as the Virgo super-cluster of galaxies can be as bright as the Moon, though only for a short time. Even so, gravitation being the weakest of all known interactions, the waves associated with it are not easily 


\section{B.S. Sathyaprakash}

detectable. Thus, though GW are produced by almost all bodies in motion, in a majority of the cases the signal strengths will be far too small to be sensed with the current detectors or those that are being built. For example, our only evidence for the emission of GW comes from the observation of the decay in the orbital period of the HulseTaylor binary caused by radiation back-reaction []. Yet the wave amplitude (and the GW frequency) is far too small to be sensed by any antenna - existing, under construction or planned. Only a catastrophic astronomical event can lead to a wave amplitude high enough to be detectable.

In general relativity the effects of both the gravitational interaction and GR are described by the tidal distortion they produce in the distance between pairs of free test masses; the Newtonian inverse-square force field simply determines the background geometry in which free particles move along geodesics. The Coulomb tidal force due to a gravitating system falls off as $r^{-3}$, whereas the tidal force due to GW that it emits falls off as $r^{-1}$. Thus, the gravitational interaction is the dominant effect close to a gravitating body and the effect of the waves is pretty much negligible. However, far away from the body the effect of the waves are more easily sensed than the Coulomb interaction.

There is a fundamental difference in the way GW sources are observed as opposed to electro-magnetic wave sources: Optical, infrared, radio and other telescopes are essentially sensitive to the intensity of radiation. They build up the signal-to-noise ratio (SNR) by incoherent superposition of waves emitted by a large number of microscopic sources. One, therefore, only obtains a gross picture of the region emitting the radiation. The intensity falls off with distance as $r^{-2}$ so that the number of sources accessible to a telescope that is sensitive to sources with a limiting intensity $I_{0}$, increases as $I_{0}^{-3 / 2}$, as the limiting intensity decreases. The frequency of electro-magnetic radiation is rather large, $10^{8}-10^{18} \mathrm{~Hz}$ and one normally deals with a narrow-band detector.

In contrast to astrophysical electro-magnetic radiation, all GW signals, with the exception of stochastic backgrounds and signals of unknown shape, are tracked in phase and the SNR is built up by coherent superposition of many cycles emitted by a source. In this case it is possible to deduce the detailed dynamics of the bodies emitting radiation and a precise picture of the source can be reconstructed. Since one tracks the phase of a signal, the SNR is proportional to the amplitude and only falls off as $r^{-1}$ with distance. Therefore, the number of sources accessible to a GW detector that is sensitive to sources with a limiting amplitude $h_{\min }$, increases as $h_{\min }^{-3}$, as $h_{\min }$ decreases. Thus GW detectors can quickly improve their sensitivity and begin to see sources at the edge of the Universe. Astrophysical and cosmological GW have a frequency range of $10^{-4}-10^{4} \mathrm{~Hz}$ and the detectors are generally quite broad band.

GR can be represented by a second rank, symmetric trace-free tensor. In a general coordinate system, and in an arbitrary gauge, this tensor has nine independent components. However, as in the electro-magnetic case, GR has only two independent states of polarisation in Einstein's theory: the plus-polarisation $h_{+}$and the cross-polarisation $h_{\times}$(the names being derived from the shape of the equivalent force fields they produce). However, unlike electro-magnetic radiation the angle between the two polarisation states is $\pi / 4$ rather than $\pi / 2$. This is illustrated in Fig. 1 where the response of a ring free particles in the $(x, y)$ plane to plus-polarised and cross-polarised $\mathrm{GW}$ traveling in $z$-direction is shown. Measuring the polarisation states is of fundamental importance since there are theories of gravity, other than general relativity, in which the number of polarisation states is more than two; in some theories even dipolar and scalar waves exist []. Polarisation measurement is a qualitative test: Even a single measurement of a polarisation state that is not plus or cross, can rule out general relativity. Such measurements would be possible with a network of detectors.

Measuring the polarisation of the waves has astrophysical implications too. Astrophysical observations can only determine the mass of a binary to within a factor $\sin \theta$ where $\theta$ is the unknown inclination angle of the binary with the line-of-sight. From GW polarisation measurements of astrophysical binaries we will be able to infer the inclination angle which will further help us to resolve the mass-inclination angle degeneracy.

\section{Gravitational wave detectors and their sensitivity}

Our detectors cannot directly measure the two independent polarisations of the waves but rather a certain linear combination of the two. The complex response $R$ of a detector is []:

$$
R=h_{+} F_{+}+i h_{\times} F_{\times}, \quad F_{+}=\Re(F), \quad F_{\times}=\Im(F),
$$

where $F_{+}$and $F_{\times}$are the real and imaginary parts of the complex antenna pattern, which is a function of the direction $(\theta, \phi)$ to the source and the polarisation angle $\psi$ of the wave, in a coordinate system 'attached' to the detector. Responses of three widely separated detectors to an incident GW, together with two independent differences in arrival times among them, are, in principle, sufficient to fully reconstruct the GW. For a resonant bar detector, with its longitudinal axis aligned with the $z$-axis, the response is,

$$
F=\sin ^{2} \theta e^{2 i \psi},
$$

and for an interferometer with its arms in the $(x, y)$ plane and at right angles to each other and the $x$-axis bisecting the two arms, 
Gravitational Wave Symphony
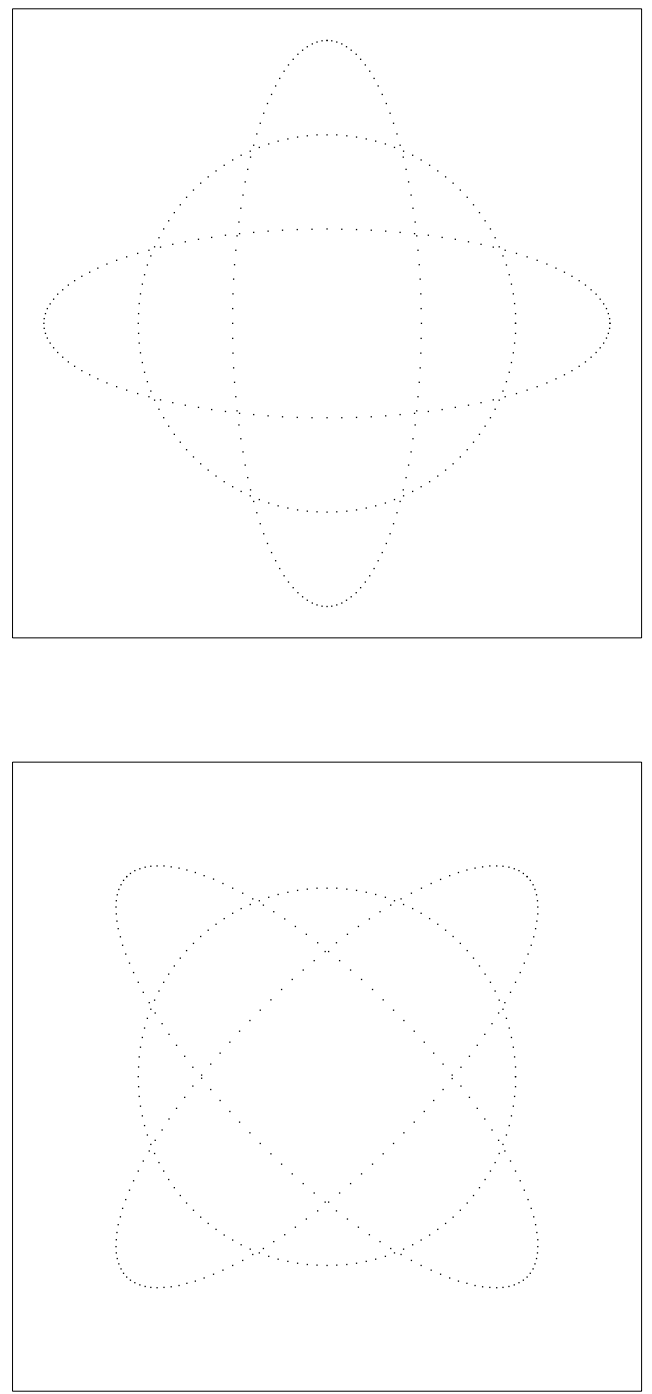

Figure 1. In Einstein's theory gravitational waves have two independent polarisations. The effect on a circular ring of particles in the $(x, y)$ plane due to a plus-polarised wave traveling in the $z$-direction is shown in the left panel and due to a cross-polarised wave is shown in the right panel. The ring continuously gets deformed into one of the ellipses and returns to the circular configuration during the first half of a GW period and gets deformed into the other ellipse and back during the next half. 


\section{B.S. Sathyaprakash}
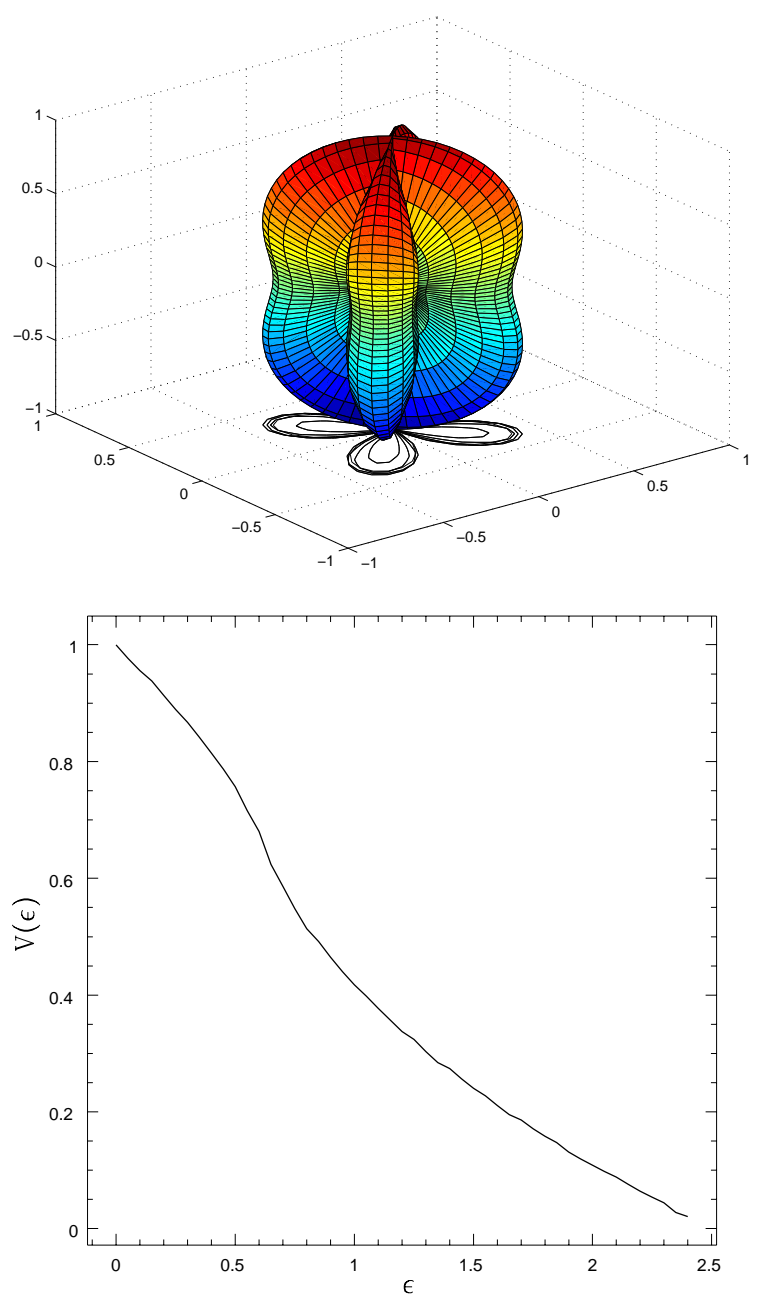

Figure 2. The antenna pattern of an interferometric detector (left panel). The pattern is shown for a circularly-polarised waves. The response for a wave traveling in a certain direction is proportional to the distance from the origin, at the centre of the plot, to the point on the antenna pattern in that direction. Also shown is the fractional area on the sky over which the response is a certain fraction $\epsilon$ of the average (right panel). The average response has been arbitrarily set to unity. 


$$
F=\left[\frac{1}{2}\left(1+\cos ^{2} \theta\right) \sin 2 \phi+i \cos \theta \cos 2 \phi\right] e^{2 i \psi} .
$$

In the following we shall assume that the two polarisation amplitudes are equal: $h_{+}=h_{\times} \equiv h / \sqrt{2}$, that is circularly polarised waves. The response of an interferometric detector is plotted in Fig. 2. The rms response of an interferometric detector, averaged over the entire sky, is smaller by a factor of $\sqrt{5}$ than the maximum response. Fig. 2. also shows the percentage area of the sky over which the response in an interferometric antenna is larger than a certain fraction $\epsilon$ of the rms response. The response is better than $50 \%$ of the rms over $75 \%$ of the sky, implying that interferometric detectors are quite omni-directional. In contrast, the rms response of a resonant bar is smaller by a factor of $1 / \sqrt{15}$ than the optimum response and the response is better than $50 \%$ over $69 \%$ of the sky. Thus, bars too are almost as omni-directional as interferometers.

Currently, there are a number of bar detectors in operation: some of these operate at room-temperature and some others at cryogenic temperatures. Bar detectors are resonant, narrow-band detectors. They can detect signal amplitudes $h \sim 10^{-20}$ in a band width of $10-20 \mathrm{~Hz}$ around a central frequency that lies at about $1 \mathrm{kHz}$. Asymmetric supernovae in our Galaxy are the best candidate sources for these detectors. They may also see continuous radiation emitted by a neutron star if the frequency happens to lie in their sensitivity band.

Interferometric detectors currently under construction will increase our ability to directly observe GW. The Japanese have already built a $300 \mathrm{~m}$ detector TAMA in Tokyo, Japan []. Several other projects are now nearing completion: The British-German collaboration is constructing a $600 \mathrm{~m}$ interferometer GEO in Hannover, Germany [], the French-Italian collaboration is building a $3 \mathrm{~km}$ detector VIRGO near Pisa, Italy [] and the Americans are building two $4 \mathrm{~km}$ antennas LIGO, one in Livingston and the other in Hanford [] in the U.S.A. These detectors will start taking data between 2001 and 2003. The larger of these detectors, LIGO and VIRGO, are likely to be upgraded in sensitivity by an order of magnitude with a better low-frequency performance in 2006. These ground based interferometers will eventually be sensitive to sources in the frequency range from $1 \mathrm{~Hz}$ to several $\mathrm{kHz}$.

One has to move to space to observe GW sources whose time periods are larger than about 1 second. Currently the prospect for constructing the Euro-American Laser Interferometer Space Antenna (LISA) looks very good []. LISA consists of three drag-free satellites, forming an equilateral triangle of size 5 million $\mathrm{km}$ and in an heliocentric orbit, lagging behind Earth by 30 degrees. LISA will be sensitive to waves in the low-frequency band of $10^{-4}-1 \mathrm{~Hz}$.

The performance of a GW detector is characterized by the power spectral density (PSD) $S_{h}(f)$ of its noise background which has dimensions of $\mathrm{Hz}^{-1}$ (see [] for a discussion on the noise PSD of interferometers). One compares the GW amplitudes of astronomical sources with the instrumental sensitivity and assesses what sort of sources will be observable in the following way: The Fourier component $\tilde{h}(f)$ of a deterministic signal $h(t)$ has dimensions of $\mathrm{Hz}^{-1}$ and the quantity $f|\tilde{h}(f)|$, is dimensionless. This is compared with $h_{n}(f) \equiv f S_{h}(f)$, the dimensionless noise PSD per logarithmic bin, to deduce the strength of a source relative to the detector noise. The noise PSD of ground- and space-based interferometric GW detectors is shown in Fig. 3 .

It is quite common also to compare a signal's amplitude spectrum per logarithmic bin, $\sqrt{f}|\tilde{h}(f)|$, with the amplitude spectrum of noise, $\sqrt{S_{h}(f)}$, both having dimensions of per root Hz. Finally, for monochromatic sources, one compares the effective noise in a long integration period with the expected "instantaneous" amplitudes as follows: A monotonic wave of frequency $f_{0}$ observed for a time $T$ is simply a narrow line in a frequency bin of width $\Delta f \equiv 1 / T$ around $f_{0}$. The noise in this bin is $S_{h}(f) \Delta f=S_{h}(f) / T$. Thus the SNR $\rho$ after a period of observation $T$ is SNR $=h_{0} / \sqrt{S_{h}\left(f_{0}\right) / T}$. One therefore computes this dimensionless noise spectrum for a given duration of observation, $S_{h}(f) / T$, to assess the detectability of a continuous GW.

\section{Sources of gravitational waves}

Inferences drawn from astronomical observations have led to the belief that luminous matter constitutes a small fraction of the total matter content of the Universe. More than $90 \%$ of the mass in the Universe is electro-magnetically silent. The presence of dark matter is inferred from the gravitational influence it causes on luminous matter. It is possible that some fraction of this dark matter is a strong emitter of GR. Today our firm sources are only those inferred via astronomical observations but it is only when we learn how to make gravitational observations of these sources can we hope to explore the unknown Universe in detector data. The reason for this is partly because we do not know what sort of radiation to expect from these plausible sources and partly because $\mathrm{GW}$ data analysis is a complex process involving 10 's of Terabytes of data each year, from hundreds of different detector channels. After an initial steep learning curve gravitational observations could prove to be a very rewarding exercise. In this Section we will take a look at some of the anticipated sources. 


\section{B.S. Sathyaprakash}
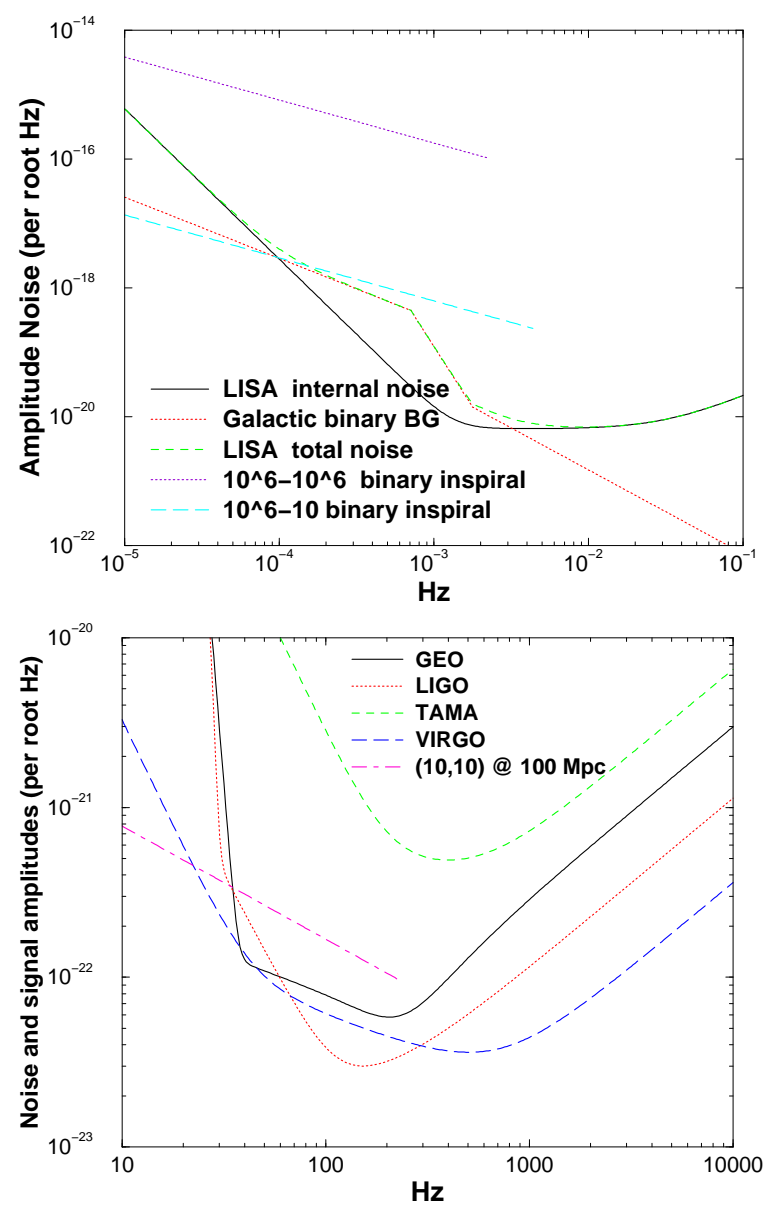

Figure 3. The amplitude noise spectral density, $\sqrt{S_{h}(f)}$, in ground- and space-based interferometers. On the same graph we also plot the signal amplitude, $\sqrt{f}|\tilde{h}(f)|$, of binary black holes - inspiral occurring at a distance of $100 \mathrm{Mpc}$ in the case of stellar mass black holes and at $3 \mathrm{Gpc}$ in the case of super-massive black holes. LISA can super-massive black hole mergers with a good SNR practically anywhere in the Universe. 


\section{Gravitational Wave Symphony}

\subsection{Gravitational wave bursts}

GW carry energy and momentum from their sources. By demanding that the energy lost in the form of GR is precisely balanced by the decrease of the energy in the system, one can derive a simple expression for the apparent luminosity of radiation $\mathcal{F}$, at great distances from the source, in terms of the GW amplitude []:

$$
\mathcal{F}=\frac{|\dot{h}|^{2}}{16 \pi}
$$

where $h$ denotes the time-derivative of the GW amplitude $h$. This equation can be used to make order-of-magnitude estimates of GW amplitudes. If a source at a distance $r$ radiates energy $E$, at a frequency $f$, in a time $T$, then writing $\dot{h}=2 \pi f h$ and $\mathcal{F}=E /\left(4 \pi r^{2} T\right)$, the amplitude is

$$
h \sim \sqrt{\frac{E}{T}} \frac{1}{\pi r f} .
$$

The amplitude of a fiducial stellar mass source that deposits a small fraction of its total mass in the form of GW over a time scale of a few milliseconds would be

$$
h \sim 4 \times 10^{-21}\left(\frac{E}{10^{-7} M_{\odot}}\right)^{1 / 2}\left(\frac{5 \mathrm{~ms}}{T}\right)^{1 / 2}\left(\frac{200 \mathrm{~Hz}}{f}\right)\left(\frac{40 \mathrm{kpc}}{r}\right) .
$$

Two masses separated by a distance of $\ell=1 \mathrm{~km}$ will be tidally distorted by no more than $4 \times 10^{-18} \mathrm{~m}$ by such a wave. This is a change in length that is smaller than the size of a neutron and a phase change of order $5 \times 10^{-10}$ radians in an interferometer that uses 0.1 micron laser and whose arms are $4 \mathrm{~km}$ long.

Long baseline interferometers, such as LIGO and VIRGO, aim towards achieving a dimensionless noise amplitude $\sqrt{f S_{h}(f)}$ of $4 \times 10^{-22}$ at about $200 \mathrm{~Hz}$ during their initial operation. From Eq.(6) we see that an event at $40 \mathrm{kpc}$, a distance covering our own Galaxy and some of its satellites, depositing energy equivalent to $10^{-7} M_{\odot}$ at $200 \mathrm{~Hz}$ in 5 ms will appear in these detectors with a maximum SNR of 10 or a sky and polarisation averaged SNR (which is $\sqrt{5}$ smaller than the maximum) of about 4.5. This is quite a good SNR for a network containing 3 or more interferometers.

The numbers we have used for energy, duration and frequency are typical of what is expected for a strong type II supernova. It is expected that an optically bright supernova event may occur as often as once every 30 to 100 years per galaxy. If for some reason gravitational collapse and the ensuing supernova, in a good fraction of these events, are not optically bright then the number of gravitationally observable events might be somewhat higher and within the region which the initial interferometers will be able to survey there may be several events per year. In any case second generation antennas should indeed observe quite a large number of these events shedding some light on the nature of the gravitational collapse. Future detectors may give us clues on the equation of state of dense nuclear matter of gravitationally collapsed stars by observing the radiation emitted as the collapsed star settles downs to a quiescent state.

\subsection{Matched filtering and effective amplitude}

Theoretical calculations sometime allow us to accurately predict the evolution of a system and the phasing of the GW that it emits. When the time development of a signal is known, one can cross-correlate the detector output with a copy of the expected signal. The process of cross-correlation leads to an enhancement in the SNR in proportion to the square-root of the number of cycles the signal spends in the detector band. Note, however, that one will have to perform the cross-correlation of the detector output with a number of different copies of the signal, each corresponding to a different set of values of the signal parameters; this process is called matched filtering. In spirit, matched filtering of arbitrary signals is the same as a simple Fourier transform of mono-chromatic signals. For illustrative purposes, therefore, we shall assume that the signal is mono-chromatic. A signal of frequency $f$ lasting for a time $T$ would produce $n=f T$ cycles. Using this in Eq. (6) we can define an effective amplitude $h_{\text {eff }} \equiv \sqrt{n} \times h$ :

$$
h_{\mathrm{eff}}=4 \times 10^{-21}\left(\frac{E}{0.05 M_{\odot}}\right)^{1 / 2}\left(\frac{200 \mathrm{~Hz}}{f}\right)^{1 / 2}\left(\frac{30 \mathrm{Mpc}}{r}\right),
$$

where we have used the energy and frequency appropriate for a stellar mass black hole binary close to its coalescence. From the above equation we conclude that matched filtering helps in detecting signals as long as the effective amplitude $h_{\text {eff }}$ is larger, even though the instantaneous amplitude $h$ could be much smaller, than the noise background. Indeed, the instantaneous amplitude can be smaller than the minimal detectable amplitude of a detector by a factor of $\sqrt{n}$. 


\section{B.S. Sathyaprakash}

The number of cycles for supernovae, black hole quasi-normal modes and other impulsive events is of order 10, for binary inspiral coalescences it can vary between 10 to $10^{4}$ and for spinning neutron stars in a year's observation it can be as large as $10^{10}$. Matched filtering can, therefore, greatly improve the visibility of signals of known shape. This is the reason why there is a lot of emphasis on studying detailed dynamics of GW sources. Of all sources, black hole binaries and normal modes of neutron stars have been studied in greatest detail.

\subsection{Binary black holes and neutron stars}

Binaries consisting of double neutron stars (NS), double black holes (BH) or a NS and a BH, are called compact binaries. Compact binaries are among the best studied and most promising sources for the upcoming interferometric GW antennas. In this Section we will discuss the prospect for detecting these systems.

\section{The evolution of a compact under radiation reaction}

Gravitational waves carry energy and angular momentum from a compact binary causing the system to eventually merge into a single black hole, emitting a burst of radiation in the process. The amplitude and frequency of the waves increase as the binary inspirals resulting in a characteristic chirping signal. Post-Newtonian (PN) theory has been used to compute the evolution of such a system but as the two stars approach each other the PN theory becomes less and less accurate, until finally, when the two stars are at a distance $r \simeq 6 M$, the PN approximation completely breaks down.

Recent advances in radiation reaction calculations [] and PN theory [] and their refinements [], have shed some light on the merger phase []. While these developments will help to some extent in tracking the inspiral and coalescence, a more careful treatment of the problem using the full Einstein equations will be needed to follow the non-linear evolution that takes place during the merger phase. The radiation emitted at very late times - called quasi-normal modes - is again well understood using black hole perturbation theory. The missing gap is the merger phase which could last several 10's of milli-seconds in the case of stellar mass binaries. The problem in the case of a stellar mass black hole falling into a super-massive black hole is possibly extremely complicated owing to the presence of spinning bodies and eccentric orbits, and we may have to wait several years before any useful solutions are found.

\section{White-dwarf binary inspiral}

Ground based detectors are not sensitive to non-compact binaries, such as a white dwarf-white dwarf pair, which do emit GW, but at a much lower frequency than where these detectors are sensitive. GW amplitude would be the greatest when the two component stars are closest. For a pair of stars each of radius $R$, the distance of closest approach is $r=2 R$. The Keplerian frequency at this distance is

$$
f_{\text {orb }}=\frac{1}{2 \pi} \sqrt{\frac{M}{r^{3}}}=0.1\left(\frac{M}{M_{\odot}}\right)^{1 / 2}\left(\frac{r}{6000 \mathrm{~km}}\right)^{3 / 2} \mathrm{~Hz},
$$

where $M$ is the total mass of the system and the radius is taken to be that of a typical white dwarf, $R=3000 \mathrm{~km}$. We see that the largest GW frequency, which is equal to twice the orbital frequency, is outside the sensitivity band of ground-based detectors. Indeed, much before the white-dwarfs begin to touch each other the tidal interaction becomes important so that only frequencies ten to hundred times smaller than the above limit can be observed. Such frequencies are within the sensitivity band of LISA. In fact, the Galactic and extra-galactic populations of white-dwarf binaries create a stochastic GW background as shown in Fig. 3 and only the nearest sources will stand above the background noise.

\section{Compact binary inspiral}

For compact binaries the adiabatic inspiral phase, when the time-scale for frequency evolution is significantly larger than the orbital time-scale, ends well before the two stars begin to touch each other. This is because the non-linear general relativistic effects will alter the dynamics of the two bodies making the adiabatic approximation and the PN theory invalid. Apart from the break down of the physical assumptions, it is, indeed, even technically impossible to continue the orbital evolution beyond the last stable orbit under the adiabatic approximation []. For a test particle in 
Schwarzschild geometry the last stable circular orbit (LSO) occurs at a distance $R_{\mathrm{lso}}=6 \mathrm{M}$, corresponding to a LSO GW frequency $f_{\text {lso }}$ given by

$$
f_{\text {lso }}=\frac{1}{6^{3 / 2} \pi M}=1.5\left(\frac{M}{2.8 M_{\odot}}\right)^{-1} \mathrm{kHz} r .
$$

Binaries more massive than a typical NS binary of $M=2.8 M_{\odot}$ end their inspiral phase at lower frequencies. The mass of a binary whose LSO frequency is the same as the frequency $f_{\text {peak }}$ at which a detector has the best effective sensitivity is given by

$$
M_{\text {optimal }}=15\left(\frac{f_{\text {peak }}}{150 \mathrm{~Hz}}\right)^{-1} M_{\odot} .
$$

Therefore, stellar mass binaries are best suited for ground-based detectors. Astronomical observations have evidence for the existence of stellar mass single black hole systems with their mass in the range 5-15 $M_{\odot}$. However, there has been no observational evidence for a double black hole system. There is a real difficulty in observing such systems astronomically as they cannot be expected to have an accretion disc, which is the source of electro-magnetic radiation in single black hole systems. Ground-based GW detectors should eventually shed light on the occurrence of black hole binaries in nature.

LISA has its peak sensitivity $f_{\text {peak }} \simeq 1.5 \times 10^{-3} \mathrm{~Hz}$ implying that binaries with a total mass $M=1.5 \times 10^{6} M_{\odot}$, are the best candidate sources. There is mounting astronomical evidence that the centre of every galaxy hosts a massive black hole of mass in the range $10^{5}-10^{9} M_{\odot}$ depending on the type of galaxy at hand []. Moreover, images of the high red-shift Universe taken by the Hubble space telescope shows that galaxies were strongly interacting in the past and that most galaxies must have undergone merger at least once in their life-time. This merger would have resulted in the coalescence of the black holes at their centres. Moreover, the dense environs of galactic centres also host many stellar mass compact objects that are captured by the central only once in a while leading to the growth of the hole's mass. LISA will be able to detect GWs emitted in the process of massive BH inspiral and merger wherever they occur in the Universe as well as stellar mass compact objects sinking into, and coalescing with, massive black holes.

The duration of an inspiral signal observed by an antenna depends on the masses of the component stars and the lower frequency cutoff of the detector sensitivity window. An antenna with a lower cutoff frequency $f_{\mathrm{L}}$ and upper frequency cutoff larger than the LSO frequency will observe the signal for a time $\tau$ given by

$$
\tau=\frac{5}{256 \eta M^{5 / 3}\left(\pi f_{\mathrm{L}}\right)^{8 / 3}}\left[1-\left(\frac{f_{\mathrm{L}}}{f_{\mathrm{lso}}}\right)^{8 / 3}\right], \quad f_{\mathrm{L}}<f_{\mathrm{lso}} .
$$

If $f_{\mathrm{L}} \ll f_{\text {lso }}$ then

$$
\begin{aligned}
\tau & \simeq 24.2\left(\frac{0.25}{\eta}\right)\left(\frac{2.8 M_{\odot}}{M}\right)^{5 / 3}\left(\frac{40 \mathrm{~Hz}}{f_{\mathrm{L}}}\right)^{8 / 3} \mathrm{~s} \\
& \simeq 1\left(\frac{0.25}{\eta}\right)\left(\frac{2 \times 10^{6} M_{\odot}}{M}\right)^{5 / 3}\left(\frac{45.4 \mu \mathrm{Hz}}{f_{\mathrm{L}}}\right)^{8 / 3} \mathrm{yr},
\end{aligned}
$$

where $M=m_{1}+m_{2}$ is the total mass and $\eta=m_{1} m_{2} / M^{2}$ is the symmetric mass ratio. For most binaries the signal will last long enough to observe only once. In other words, all inspiral signals in ground-based detectors, and most in LISA, are transient events and the question arises how many such events are we likely to observe in a certain period of time.

Estimates of rates of binary coalescences are based either on the few observed NS-NS binaries in our Galaxy or theoretical studies of compact binary formation and evolution on a computer - the so-called population synthesis. Both these estimates are highly uncertain. The estimates give a rate between $10^{-6}-10^{-5}$ NS-NS coalescences per year in each galaxy of size the same as our own Milky-way. This gives several NS-NS coalescences per year in a volume of $(300 \mathrm{Mpc})^{3}$. Black hole binaries may have similar coalescence rate densities. However, being more massive than NS-NS binaries, BH-BH binaries can be observed from greater distances and hence the number of expected BH-BH coalescences in initial interferometers is greater.

Coalescence rates involving super-massive black holes is also quite uncertain. But it is expected that each year LISA will observe 10-100 stellar mass black holes falling into super-massive black holes and several galaxy mergers. Formation of massive black holes at galactic centres has not been well understood. Observations cannot completely rule out or favour any one formation scenario. In most scenarios it should be possible to observe the GW radiation emitted in the process of formation of the hole. The newly formed black hole would emit radiation at a frequency characteristic of its mass and spin. 


\section{B.S. Sathyaprakash}

During the inspiral regime the signal will sweep as many as

$$
N_{\text {cyc }}=\frac{1}{32 \pi \eta\left(\pi M f_{\mathrm{L}}\right)^{5 / 3}} \simeq 1600\left(\frac{2.8 M_{\odot}}{M}\right)^{5 / 3}\left(\frac{40 \mathrm{~Hz}}{f_{\mathrm{L}}}\right)^{5 / 3}
$$

cycles. The energy emitted in the inspiral phase is equal to the binding energy of the system at a separation $R=6 M$ :

$$
E=-\frac{m_{1} m_{2}}{2 R}=-\frac{\eta M^{2}}{2 R}=-\frac{\eta M}{12} .
$$

Thus, in the case of equal mass binaries (i.e., $\eta=1 / 4$ ) the maximum energy emitted during the inspiral phase is about $1 / 50$ of its total mass or roughly $0.05 M_{\odot}$ for a NS-NS binary, which is what was used in equation (77). The effective amplitude at a given frequency $f$ can be obtained by noting that the distance between the two stars is related to the GW frequency via $R=\left[M /\left(\pi^{2} f^{2}\right)\right]^{1 / 3}$, and using $|E|=\eta M^{2} /(2 R)=\eta M^{5 / 3}(\pi f)^{2 / 3} / 2$, in the expression for the effective amplitude, $h_{\text {eff }}=\sqrt{E / f} /(\pi r)$ :

$$
h_{\mathrm{eff}}=\frac{\eta^{1 / 2} M^{5 / 6} f^{-1 / 6}}{\pi^{2 / 3} r} .
$$

The above equation gives,

$$
\begin{aligned}
h_{\mathrm{eff}} & =3 \times 10^{-21}\left(\frac{30 \mathrm{Mpc}}{r}\right)\left(\frac{\eta}{0.25}\right)^{1 / 2}\left(\frac{M}{2.8 M_{\odot}}\right)^{5 / 6}\left(\frac{1 \mathrm{kHz}}{f}\right)^{1 / 6} \\
& =4 \times 10^{-17}\left(\frac{1 \mathrm{Gpc}}{r}\right)\left(\frac{\eta}{0.25}\right)^{1 / 2}\left(\frac{M}{10^{6} M_{\odot}}\right)^{5 / 6}\left(\frac{1 \mathrm{mHz}}{f}\right)^{1 / 6} .
\end{aligned}
$$

The SNR for inspiral events in various ground- and space-based interferometers are plotted for stellar mass and supermassive sources located at a fixed distance of $100 \mathrm{Mpc}$ and $3 \mathrm{Gpc}$ from Earth, respectively. The initial network of ground-based antennas have a fair chance of making the first observations of GW by tracking the inspiral signals from stellar mass black holes, while LISA can observe neutron stars and small black holes falling into super-massive black holes from up to a red-shift of 1. Galaxy mergers and the associated super-massive black hole coalescences will appear in LISA as very luminous events wherever they occur in the Universe.

\section{Binary black holes as a probe of physics, astrophysics and cosmology}

Inspiral events are wonderful test beds for general relativity, for both its fundamental predictions as well as for testing the theory in the strongly nonlinear regime. The post-Newtonian expansion of the inspiral signal has unfolded a rich cache of non-linear physics that governs the dynamics of a binary. There are many interesting effects such as GW tails, spin-spin interaction, Lens-Thirring effect, etc., which can all be deciphered from a high SNR event. For instance, the effect of tails on the phasing of GW can be detected in the inspiral waves emitted by BH-BH binaries [].

Following an inspiral event with electro-magnetic observation, such as gamma- or X-ray observation, would help to deduce the speed of GW to a phenomenal accuracy. (Even a day's delay in the arrival times of gravitational and electro-magnetic radiation from a source at a distance of million light years would determine the relative speeds to better than one part in $10^{8}$ ). This will require a good timing accuracy to determine the direction to the source so that astronomical observations of the same source can be made.

One can tighten the limit on the mass of the graviton by observing the inspiral waves. Massive gravitons would alter the phasing of the signal. Matched filtering facilitates an accurate measurement of the phasing which in turn allows to place a bound on graviton's mass. Will estimates that one can bound the mass of the graviton to $2.5 \times 10^{-22} \mathrm{eV}$ using ground-based detectors and $2.5 \times 10^{-26} \mathrm{eV}$ using space-based detectors [].

Inspiral waves are standard candles in the sense that by measuring their luminosity we can infer the distance to source Schutz []. In the quadrupole approximation, the wave's amplitude (as well as its phase) depends only on a certain combination of the masses called the chirp mass, $\mathcal{M}=\eta^{3 / 5} M$, and the distance $r$ to the source. If we can measure the amplitude and the chirp mass independently, then we can infer the distance to the source directly. Inspiral waves are detected by using matched filtering technique which enables an accurate estimation of the masses of the component stars. In addition, the SNR enables us to measure the signal's amplitude as well. Thus, inspiral waves serve as standard candles. This will allow us to determine the Hubble constant very accurately by identifying the host galaxy and measuring its red-shift. It should be noted that the inspiral wave's phase is quite sensitive to postNewtonian corrections and when they are included the mass-degeneracy is lifted. It is, therefore, possible to determine both the masses of a binary by using post-Newtonian search templates. 
Gravitational Wave Symphony
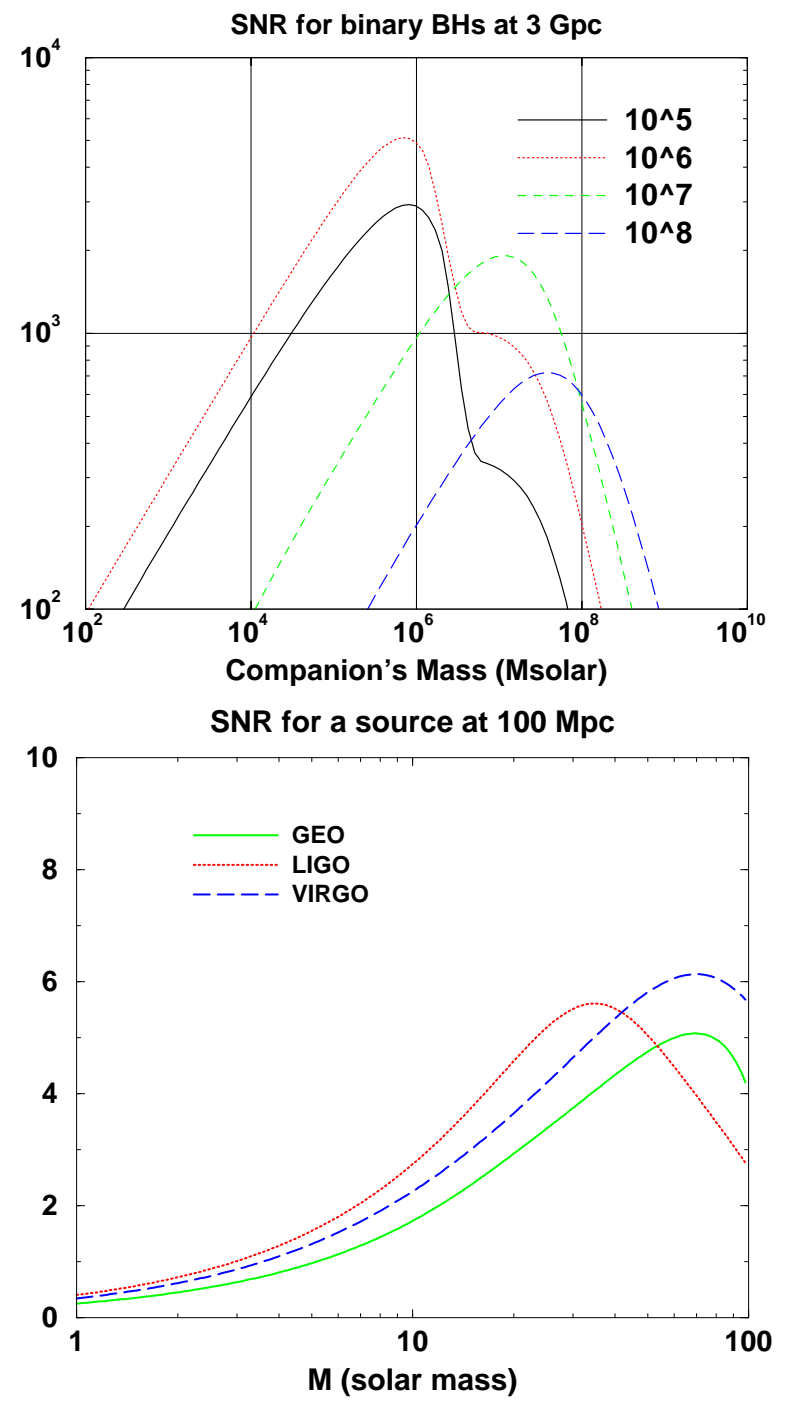

Figure 4. The expected SNR from an inspiral event is plotted for ground- and space-based interferometers. In the left hand panel we show the SNRs as a function of the companion's for different masses of central black hole. For ground-based interferometers we show the SNR as a function of the total mass of equal mass binaries but for different detectors. 


\section{B.S. Sathyaprakash}

Observations of massive black hole coalescences at cosmological distances $z \sim 1$ by space-based detectors can facilitate an accurate determination of the distance to the source. Space-based detectors observe a massive binary inspiral for a whole year and they have, thus, the baseline of the Earth's orbit around the Sun to triangulate the source on the sky. They can do this to an accuracy of about a square degree. At a distance of $3 \mathrm{Gpc}$ one square degree is roughly $100 \mathrm{Mpc}^{2}$, a scale over which no more than one virialised galaxy cluster can be found. Thus, an optical identification of the host galaxy cluster and its red-shift should be possible, which would enable the measurement of the deceleration parameter $q_{0}$, and hence the density parameter $\Omega$, of the Universe. A single source at $z \gtrsim 1$ is enough to measure both $H_{0}$ and $\Omega$ to an accuracy of better than $1 \%$.

Galaxy interactions and mergers must have been quite a common event in the early history of galaxy formation. We know very little about the history of galaxy formation and have no clue how super-massive black holes may have formed. LISA will be able to detect galaxy mergers practically anywhere in the Universe thereby helping in resolving many puzzling questions about super-massive black holes.

By measuring GWs emitted during the inspiral of a stellar mass compact object into a super-massive black hole we will be able to map the spacetime geometry around massive, rapidly spinning holes and test the uniqueness of the Kerr solution, observe precessions of the periastron and of the orbital plane and associated non-linear effects. Such observations will undoubtedly help us understand the complicated dynamics and possibly even chaotic behaviour, expected in the evolution of such systems. Thus, space-based detectors can potentially contribute quite a lot to further our understanding of fundamental science and cosmology.

\subsection{Black hole quasi-normal modes}

A compact binary coalescing as a result of gravitational radiation reaction would most likely result in the formation of a single black hole. The newly formed hole will initially be somewhat non-spherical, and this dynamical nonsphericity will be radiated away in the form of GW. The late time behaviour of this radiation is well studied in the black hole literature and there are detailed calculations of the (quasi) normal modes for both static, i.e. Schwarzschild, and stationary, i.e. Kerr, black holes. In all cases the time-evolution of the emitted radiation is well-modelled by a quasi-periodic signal of the form

$$
h(t ; \tau, \omega)=A e^{-t / \tau} \cos (\omega t)
$$

where $\tau$ is the decay time-scale of the mode in question and $\omega$ is the angular frequency of the mode, both of which depend on the black hole's mass and spin. In all but the extreme Kerr black holes (extreme Kerr black holes are those that are spinning at the maximum possible rate) the only dominant mode, i.e. the mode for which the decay time is the longest and the amplitude is the highest, is the fundamental mode whose frequency is related to the mass $M$ and spin $a$ of the black hole via $\omega=\left[1-0.63(1-a)^{0.3}\right] / M$ where $M$ is the mass of the black hole in units $G=c=1$ and $a=J / M$ is the spin angular momentum of the hole in units of black hole mass. The decay time $\tau$ is given by $\tau=4 /\left[\omega(1-a)^{0.45}\right]$. (See Ref. [] and references therein for details.)

It is estimated [] that during the quasi-normal mode ringing of a black hole the energy emitted might be as large as 3\% of the system's total mass. By matched filtering it should be possible to detect quasi-normal modes, in initial interferometers, from black holes of mass in the range $60-10^{3} \mathrm{M}_{\odot}$ and at a distance of $200 \mathrm{Mpc}$. Binary black hole mergers should result in the emission of such a ring down signal during the late stages. Thus, inspiral signals emitted before the merger might aid in identifying the quasi-normal modes.

\subsection{Continuous waves}

Our Galaxy is expected to have at least $10^{8}$ spinning neutron stars that form roughly at a rate of one every 30 years. Some population of neutron stars is in binaries. There are a number of ways in which a single spinning neutron star could radiate away GW (if the neutron star is axisymmetric, of course, then there will be no GW emission): (1) Neutron stars normally spin at high rates (several to $500 \mathrm{~Hz}$ ) and this must induce some equatorial bulge and flattening of the poles. The presence of a magnetic field may cause the star to spin about an axis that is different from the symmetry axis leading to a time-varying quadrupole moment. (2) The star may have some density inhomogeneities in the core/crust set up during its formation and/or subsequent convectively unstable motions of the core. (3) The presence of an accretion disc, with its angular momentum not necessarily aligned with that of the neutron star, can potentially alter axisymmetry. That and electromagnetic radiation reaction torques can induce and sustain wobble. (4) The normal modes of the neutron star fluid (radial and other oscillations) can extract rotational energy and re-emit in the form of GW. (5) There are certain classical and relativistic instabilities in the neutron star fluid which may cause the star to radiate away energy in the form of GR. In what follows we will only discuss a sample of recent work on the radiation from spinning neutron stars. 


\section{GW amplitude from spinning asymmetric neutron stars}

If $I_{z z}$ is the moment of inertia about the spin axis of a neutron star emitting GW at a frequency $f$ then the gravitational amplitude at a distance $r$ is:

$$
h=3 \times 10^{-27}\left(\frac{10 \mathrm{kpc}}{r}\right)\left(\frac{I_{z z}}{10^{45} \mathrm{~g} \mathrm{~m}^{2}}\right)\left(\frac{f}{200 \mathrm{~Hz}}\right)^{2}\left(\frac{\epsilon}{10^{-6}}\right),
$$

where $\epsilon$ is the ellipticity of the star. In a simple model of an equatorial plane of elliptical cross section with semi-major axis $a_{1}$ and semi-minor axis $a_{2}$, the ellipticity is $\epsilon \equiv 1-a_{2} / a_{1}$. The ellipticity is an unknown but one can obtain an upper limit on it by attributing the observed spin-down of pulsars $\dot{P}$ to gravitational radiation back reaction, namely that the change in the rotational energy $E=I \Omega^{2} / 2$ is equal to GW luminosity. Then, the ellipticity is related to the spin-down rate of a pulsar via

$$
\epsilon=5.7 \times 10^{-6}\left(\frac{P}{10^{-2} \mathrm{~s}}\right)^{3 / 2}\left(\frac{\dot{P}}{10^{-15}}\right)^{1 / 2} .
$$

Since one knows the observed values of $P$ and $\dot{P}$ one can obtain an upper limit on $\epsilon$ using the above equation. Following this method one finds that for the Crab pulsar $\epsilon \leq 7 \times 10^{-4}$. The gravitational wave amplitude corresponding to this ellipticity is $h \leq 10^{-24}$. Noting that Crab has a spin frequency of $25 \mathrm{~Hz}$ (GW frequency of $50 \mathrm{~Hz}$ ) equation (16) implies an effective amplitude $h_{\mathrm{eff}}=4 \times 10^{-20}$ after a year's integration and hence observable in initial interferometers. []. It is unlikely that the ellipticity is so large and hence the effective GW amplitude is probably much less. Yet, the prospect of seeing Crab at a tenth, or even a hundredth, of this ellipticity is quite good with first/second generation interferometers.

\section{Relativistic instabilities in young neutrons stars}

It has long been known that the fundamental mode of a neutron star fluid, undergoes unstable, as a result of GW emission [], when the spin frequency of the star is larger than a certain critical spin frequency. The physics behind this instability can be understood in the following manner: Imagine exciting a mass-quadrupole mode - that is a nonuniform distribution of mass - in a non-spinning star. The mass inhomogeneity will travel on the surface of the star at a fixed speed relative to the star and this dynamical asymmetry will cause the star to radiate GW. Gravitational waves drain the angular momentum from the modes as a result of which the modes will decay. Now consider a spinning neutron star in which a co-rotating and a counter-rotating modes are excited. For spins smaller than the pattern speeds both these modes will decay in course of time by losing angular momentum: co-rotating modes lose positive angular momentum and counter-rotating modes lose negative angular momentum. But when the neutron star spins at a rate greater than a certain critical rate, to an external inertial observer both modes will appear to be corotating. Therefore, the mode counter-rotating relative to the star will also emit positive angular momentum, causing the angular momentum associated with the mode to enhance, or for the amplitude of the mode to increase. In other words, a mode counter-rotating relative to the star, but seen co-rotating relative to the inertial observer, can only emit positive angular momentum which causes its own angular momentum to increase. The energy for this increase is supplied by the spin angular momentum of the neutron star. Thus, while the mode co-rotating with the star's spin will decay, the mode counter-rotating with the spin will grow in amplitude and emit more and more radiation. This will go on until the mode has sucked out enough spin angular momentum of the star to make the counter-rotating mode appear to be counter-rotating with respect to an inertial observer too.

It is suspected that the CFS instability will not work in the presence of viscosity and hence it may be unimportant in old neutron stars. However, newly born neutron stars will be very hot and viscous forces may be insignificant in them. Recently, another class of modes called $r$-modes have been discovered, [] which - unlike the CFS modes that are mass-quadrupole moments - are current-quadrupole moments that are unstable at all spin frequencies of the star. The physics of r-modes in neutron stars is rather too complicated and there is as yet no definitive statement about the role of these modes in GW emission in young neutron stars.

\subsection{Stochastic backgrounds}

Catastrophic processes in the early history of the Universe, as well as the myriad astrophysical sources distributed all over the cosmos, generate stochastic background of GW. Astrophysical backgrounds carry the signatures of their 


\section{B.S. Sathyaprakash}

sources. By studying the spectrum of the background it should be possible to infer the underlying population. One hopes to take a census of compact objects distributed over astronomical, and even cosmological distances, by studying these backgrounds.

A cosmological background should have been created in the very early Universe and later amplified, as a result of parametric amplification, by its coupling to the background gravitational field []. Imprint on such a background are the physical conditions that existed in the early Universe as also the nature of the physical processes that produced the background. Observing such a background is therefore of fundamental importance as this is the only way we can ever hope to directly witness the birth of the Universe. The 2.7 degree $\mathrm{K}$ microwave background, which is our firm proof that the Universe was born from that explosive event, is the relic radiation from that even but it was in equilibrium with the rest of matter for nearly 300,000 years after big bang and is freely traveling to us only since then. The GW background, on the other hand, de-couple from the rest of matter soon after the big bang, and is therefore carrying uncorrupt information about the condition of the Universe at its formation. In any realistic model of the early Universe the energy density in the cosmological background is only a very small fraction of the critical density of the Universe, indeed less than one part in $<10^{15}$. As a result it is unlikely that ground-based detectors will see this relic radiation. The present design of LISA will also not enable us to see this background and one may have to fly a special space mission tailored to the observation of this relic radiation. The science output from such an observation is so fundamental that it is well worth the effort.

Phase transitions in the early Universe, inspired by fundamental particle physics theories, and cosmological strings and domain walls are also sources of a stochastic background. These processes are expected to generate a background which has a different spectrum and strength than the primordial one. It is expected that some of these backgrounds may contribute as large as $10^{-9}$ of the closure density of the Universe. Future ground-based detectors will achieve good enough sensitivity to measure the background at the level of $10^{-9}-10^{-8}$ of the closure density. Such measurements will prove to be a good test bed for these cosmological models.

\section{Acknowledgements}

I would like to thank Sayan Kar and Naresh Dadhich for invitation to and hospitality at the International Conference on Gravitation and Cosmology held at IIT Kharagpur. Without Sayan's insistence this review wouldn't have been completed; to that I am indebted to him.

\section{References}

[1] J.H. Taylor, Rev. Mod. Phys. 66, 711 (1994).

[2] B.F. Schutz, Class. Quantum Grav. 16 A131, (1999) gr-qc/9911034

[3] K.S. Thorne, in Proceedings of Snowmass 1994 Summer Study on Particle and Nuclear Astrophysics and Cosmology, E.W. Kolb and R.D. Peccei, Eds., (World Scientific, Singapore, 1995) pp 160-184.

[4] É.É. Flanagan, Astrophysical sources of gravitational radiation and prospects for their detection, in Proceedings of 15th International Conference on General Relativity and Gravitation (GR15), eds N. Dadhich and J.V. Narlikar (IUCAA, Pune, India, 1998); gr-qc/9804024

[5] L.P. Grishchuk, V.M. Lipunov, K.A. Postnov, M.E. Prokhorov, B.S. Sathyaprakash, Gravitational Wave Astronomy: In Anticipation of First Sources to be Detected, Phys. Usp. (in press) astro-ph/0008481

[6] J.-A. Marck and J.-P. Lasota, eds Relativistic gravitation and gravitational radiation (Cambridge Univ. Pr., Cambridge, 1997).

[7] B.R. Iyer and B. Bhawal, Black holes, gravitational radiation and the universe, (Kluwer Academic Press, 1998).

[8] C.M. Will, Theory and Experiment in Gravitational Physics (Cambridge Univ. Press, Cambridge, 1981).

[9] B.F. Schutz, and Tinto, M. (1987) Mon. Not. R. Astron. Soc. 224, 131.

[10] S.V. Dhurandhar and M. Tinto, Mon. Not. R. Astron. Soc. 234, 663 (1988).

[11] B.F. Schutz, in A First Course in General Relativity (Cambridge University Press, Cambridge, 1985).

[12] H. Lück et al., Class. Quantum Grav. 14, 1471 (1997).

[13] A. Abramovici et al., Science 256, 325 (1992).

[14] B. Caron et al., Class. Quantum Grav. 14, 1461 (1997).

[15] K. Tsubono, in First Edoardo Amaldi Conference on Gravitational Wave Experiments, (Singapore: World Scientific, 1995) p. 112.

[16] Bender, P. et al. LISA: Pre-Phase A Report, MPQ 208 (Max-Planck-Institut für Quantenoptik, Garching, Germany). (Also see the Second Edition, July 1998.)

[17] D. Hils, P.L. Bender, R.F. Webbink, Astrophys. J. 36075 (1990).

[18] T. Damour and N. Deruelle, Phys. Lett. 87A, 81 (1981); and C.R. Acad. Sci. Paris 293 (II) 537 (1981); T. Damour, C.R. Acad. Sci. Paris 294 (II) 1355 (1982); and in Gravitational Radiation, ed. N. Deruelle and T. Piran, pp 59-144 (North-Holland, Amsterdam, 1983). 
[19] L. Blanchet, T. Damour, B.R. Iyer, C.M. Will and A.G. Wiseman, Phys. Rev. Lett. 74, 3515 (1995); L. Blanchet, T. Damour and B.R. Iyer, Phys. Rev. D51, 5360 (1995); C.M. Will and A.G. Wiseman, Phys. Rev. D54, 4813 (1996); L. Blanchet, B.R. Iyer, C.M. Will and A.G. Wiseman, Class. Quantum Grav. 13, 575, (1996); L. Blanchet, Phys. Rev. D54, 1417 (1996);

[20] T. Damour, B.R. Iyer, B.S. Sathyaprakash, Phys. Rev. D57, 885 (1998); ibid 62, 084036 (2000);

[21] A. Buonanno and T. Damour, Phys. Rev. D59, 084006 (1999); ibid, 62, 064015 (2000).

[22] T. Damour, B.R. Iyer, B.S. Sathyaprakash, Phys. Rev. D (in press); gr-qc/0010009.

[23] C.M. Will, Phys. Rev. D57, 2061 (1998).

[24] L. Blanchet and B.S. Sathyaprakash, Class. Quantum Grav. 11, 2807 (1994); ibid, Phys. Rev. Lett. 74, 1067 (1995).

[25] B.F. Schutz, Nature 323, 310 (1986).

[26] M.J. Rees, Class. Quantum Grav. 14, 1411 (1997).

[27] F. Echeverria Phys. Rev. D40 3194 (1989).

[28] É.É. Flanagan and S. Hughes, Phys. Rev. D57, 4535 (1998).

[29] S. Chandrasekhar, Phys. Rev. Lett. 24611 (1970); J.L. Friedman and B.F. Schutz, Astrophys. J. 222281 (1978).

[30] N. Andersson, Astrophys. J. 502708 (1998);

[31] L. Lindblom, B.J. Owen and S.M. Morsink Phys. Rev. Lett. 804843 (1998).

[32] N. Andersson and K. Kokkotas, Int. J. Mod. Phys. (in press); gr-qc/0010102.

[33] B. Allen in Ref. [6]. 